\title{
Qeauty and the Books: A Response to Lewis's Quantum Sleeping Beauty Problem
}

\author{
Daniel Peterson*
}

June 2, 2009

\begin{abstract}
In his 2007 paper "Quantum Sleeping Beauty", Peter Lewis poses a problem for appeals to subjective probability in the Everett interpretation of quantum mechanics. Lewis's argument hinges on parallels between the traditional "sleeping beauty" problem in epistemology and his quantum variant. These two cases, Lewis argues, treat probability differently despite the fact that they share important epistemic similarities, thus leading to a tension between the traditional solution to the sleeping beauty problem (typically called the "thirder" solution) and Everettian quantum mechanics. My purpose in this paper is to examine the metaphysical and epistemological differences between these two cases and, on this basis, determine if Lewis's argument for a troubling tension between the Everettian and the thirder is as powerful as Lewis suggests. In particular, I examine the way in which diachronic Dutch book arguments suggest that the thirder solution in the traditional case is correct while the Everettian's solution in the quantum case is correct. These Dutch book arguments, I argue, reveal an important disanalogy between the two cases, and thus Lewis's argument does not reveal an inconsistency in either the Everettian's or the thirder's treatment of probability.
\end{abstract}

\section{Introduction}

The traditional "sleeping beauty" problem goes as follows: consider a person (for convention's sake, call her "Beauty") who is the subject of a rather bizarre experiment. Beauty is put to sleep on Sunday night, after which her experimenters flip a coin. If the coin lands on heads, Beauty is awakened on Monday and on Tuesday; however, after waking on Monday, a memory drug is administered to Beauty to make her forget this waking. If the coin flip is heads, then when Beauty awakes on Tuesday (her second awakening), she does not remember having ever been awakened before. If the experimenters' coin comes up tails, Beauty is awoken just once, on Tuesday.

The question of interest is what credence (i.e. degree of belief) Beauty should rationally assign to the proposition "the coin landed on tails" upon awaking. As Peter

\footnotetext{
*Department of Philosophy, University of Michigan, Ann Arbor, MI 48109; petersod@umich.edu
} 
Lewis points out in his "Quantum Sleeping Beauty" paper, the dominant answer to this question in the sleeping beauty literature is "one third", as argued for by Elga (2000), Arntzenius (2002), and Dorr (2002) among others. There are those who support the "one half" answer as well, most notably David Lewis (2001), but by far the most popular intuition is that Beauty should have a credence of one third. I examine what I consider to be the most persuasive argument for this "thirder" solution in the next section, but a number of traditional thirder arguments run as follows: when Beauty awakes, she has lost herself among three different outcomes: Heads/Monday, Heads/Tuesday, and Tails/Tuesday. Elga argues that, if Beauty learns it is Tuesday, she is indifferent as to whether the coin flip was heads or tails, and if she learns the coin flip came up heads, she is indifferent as to whether it's Monday or Tuesday. Thus, Elga argues, all three outcomes should carry the same credence, and so Beauty should be a thirder. Halfers like Lewis respond that Beauty learns no new information when she wakes up: she knew she was going to wake up at least once no matter what the outcome. Thus, when Beauty updates her credences using Bayes' theorem, she finds that she should have the same credence upon awaking that she had before going to sleep. Though halfer arguments like Lewis's have won their supporters, Peter Lewis points out that most philosophers tend to favor the thirder position.

Peter Lewis uses the near-consensus on the sleeping beauty problem to raise an objection against the Everettian interpretation of quantum mechanical probabilities. Put simply, the Everett or "many worlds" interpretation of quantum mechanics holds that, upon measurement, a quantum mechanical superposition of a spin-up and spin-down electron, for instance, splits into two branches, one of which is characterized by a spinup electron and one of which is characterized by a spin-down electron. Many opponents of Everett have claimed that the Everett interpretation cannot make sense of quantum mechanical probabilities; however, recent discussions of Everettian probability, such as those found in Greaves (2007), take subjective probability and decision-theoretic axioms as central to a fully Everettian account of probability. By embracing subjective probabilities, Everettians have found a way to talk about probabilities in quantum mechanics that avoids the pitfalls of previous Everettian attempts; however, for Peter Lewis, at least, such an application of subjective probabilities leads to problems for the Everett interpretation.

Peter Lewis sees parallels here between the way in which Beauty's possible-worlds successors are related to one another and the way in which an agent's many-worlds successors are related to one another in the Everett interpretation. Lewis asks us to consider three time-slices of Beauty in a world where the coin comes up "heads": one, the time-slice at $t_{0}$, is Beauty before she goes to sleep. The second time-slice, at $t_{1}$, is Beauty when she is awoken on Monday, and the third time-slice, at $t_{2}$, is Beauty when she is awoken on Tuesday. Lewis points out that though Beauty at $t_{0}$ is psychologically continuous with Beauty at $t_{1}$ and Beauty at $t_{2}$, these two successors are barred from being psychologically continuous with one another by the memory drug administered to Beauty after each awakening. Likewise, in a two-branch Everettian case an agent can be treated as having a successor in each of the two post-measurement worlds which are psychologically continuous with the agent before the branching even though neither successor is psychologically continuous with the other successor.

The similarity between an agent's successors in the Everettian case and Beauty's 
time-slices in the sleeping beauty problem leads Lewis to posit that the rational assignment of credences (i.e. subjective probabilities) in both cases should agree. Lewis states, "given the parallels between the two cases, then all other things being equal, we should expect the two cases to be governed by a uniform account of probability" (62). Lewis goes on to show that such an account cannot be achieved. I present a variant of the case Lewis examines in his paper to show how the sleeping beauty and "quantum sleeping beauty" cases differ ${ }^{1}$.

Consider Beauty's sister Qeauty, who participates in an experiment similar to Beauty's. The experimenters put Qeauty to sleep on Sunday and flip a coin. If the result of the flip is "heads", they do an x-spin measurement on an electron in a superposition of $\mathrm{x}$-spin up and $\mathrm{x}$-spin down (with probabilities of one half corresponding to each outcome per the Born rule). If the result of the measurement is $\mathrm{x}$-spin up, they wake up Qeauty up on Monday then put her back to sleep. If the result of the measurement is X-spin down, they wake her up on Tuesday and then put her back to sleep. Finally, if the coin lands on tails, the experimenters wake Qeauty up on Tuesday, then put her back to sleep.

Assuming that Qeauty is an Everettian, her case mirrors her sister's in many ways. First of all, Qeauty in a world where heads is flipped is similar to Beauty in a world where heads is flipped by Lewis's lights: Qeauty has two successors here, both of whom are psychologically continuous with pre-branching Qeauty but, by the Everettian's lights, are not psychologically continuous with each other. Likewise, Beauty has two post-Sunday successors, both of whom are psychologically continuous with Sunday Beauty but which are not psychologically continuous with each other because of the forgetfulness drug. Finally, as in the Beauty case, Qeauty has self-locating uncertainty: that is, she may be on one of many branches, yet when she wakes up, she does not know which branch she is on. Given the many similarities between the Beauty and Qeauty cases, Lewis's claim that the same account of probability should govern both cases seems reasonable.

If Lewis is right, one would expect that the Everettian's account of subjective probability in the Qeauty case should agree with the consensus about credences in the Beauty case. However, Lewis points out that this expectation is not met. The assessment of probability supported by Everettians, Lewis claims, requires that one take the halfer position to be the correct one in the Qeauty case; an Everettian Qeauty will treat her situation as if the probability of being awoken on Monday and the coin being heads is the same as the probability of flipping heads twice on a fair coin, or one quarter ${ }^{2}$. An Everettian Qeauty will thus treat the probability of the coin landing on heads as equal to the probability of the coin landing on tails, which entails the halfer solution. Since the popular solution to the Beauty case is the thirder solution, Lewis's expectation that the credences in these two cases should mirror each other is clearly violated. Lewis states that he would be content with such divergence if the two cases were obviously disanalogous, but, since the epistemic similarity between Beauty's and Qeauty's successors in the two cases leads us to believe that the two cases should

\footnotetext{
${ }^{1}$ I should note that what I call the Quantum Sleeping Beauty problem or case is one of several problems that Lewis presents in his paper; however, I take it that, should my response to the case I pose in this paper be sufficient, an analogous solution will be available as a response to Lewis's other problems.

${ }^{2}$ Lewis (2007), pp 63.
} 
treat probability the same way, the divergence seems to indicate a problem either with the logic of the thirder Beauty or of the halfer Qeauty. Lewis's own position is that this inconsistency poses a problem primarily for the Everettian, and so his "quantum sleeping beauty" argument gives us a reason to reject the Everettian's account of probability.

Papineau and Dura-Vila (2009) raise some issues with Lewis's argument, primarily arguing that there are important metaphysical differences between the Beauty and Qeauty cases that Lewis fails to acknowledge, and thus Lewis is wrong to expect the two cases to treat probability analogously. I agree with Papineau and Dura-Vila that there is an important distinction between Beauty's case and Qeauty's case that needs to be taken into account, but I do not think that Papineau and Dura-Vila's metaphysical charges are specific or detailed enough to pose much of a threat to the quantum sleeping beauty argument. My purpose in this paper is to examine the metaphysical and epistemological differences between the Beauty and Qeauty cases and, on this basis, determine if Lewis's argument for a troubling tension between the Everettian and the thirder is as powerful as Lewis suggests. In particular, I examine the way in which diachronic Dutch book arguments suggest that the thirder solution to the Beauty case is correct while the halfer solution in the Qeauty case is correct. These Dutch book arguments, I argue, reveal an important disanalogy between the two cases, and thus Lewis's argument does not reveal an inconsistency in either the Everettian's or the thirder's treatment of probability.

\section{Beauty, Qeauty, and Dutch Books}

In his "Beauty and the Bets", Hitchcock provides what I take to be the strongest argument in favor of the thirder position for the Beauty case. Hitchcock argues that a diachronic Dutch book can be made against the halfer in the traditional Sleeping Beauty case even when strict conditions are imposed on the Dutch bookie in this scenario. Dutch books reveal incoherent credences at play; for instance, if my credences require that I have a credence of both one half and one quarter in the same outcome, a Dutch book argument may be constructed against me. One can thus, like Hitchcock, take the existence of a Dutch book against a position as a reason to accept its alternatives. What follows is a basic reconstruction of Hitchcock's argument, which shows how such a book may be constructed in Beauty's case.

Hitchcock first proposes a Dutch book that could be made against the thirder. The bets in this book exploit the fact that the thirder position violates the reflection principle: that is, according to the thirder, Beauty should take the probability of a tails on the coin flip to be one half before going to sleep but one third upon waking up. The halfer could thus propose the following two bets, the first of which is taken by Beauty before falling asleep and other of which is taken by Beauty after she wakes up:

Bet 1 (before sleep): $\$ 30$ if tails, nothing if heads. Cost: $\$ 15$

Bet 2 (upon awaking): $\$ 30$ if heads, nothing if tails. Cost: $\$ 20$

A thirder Beauty will find both of these bets acceptable at the time they are suggested: 
her credence in tails at the time of the first bet is one half, and her credence in heads at the time of the second bet is two thirds. However, if she accepts these bets, she loses money. If the coin comes up heads, she receives $\$ 30$ from Bet 2 and $\$ 0$ from Bet 1 , which is not enough to offset the $\$ 35$ she paid for the two bets. Likewise, if the coin comes up tails, she receives $\$ 30$ from Bet 1 and $\$ 0$ from Bet 2, which is not enough to offset the $\$ 35$ she paid for the two bets. Thus, she loses $\$ 5$ no matter what, and these bets constitute a Dutch book.

Not so fast, Hitchcock says. One of the crucial assumptions made concerning the bookie in these diachronic Dutch book cases is that he has no more information than the book-ee has ${ }^{3}$. While this condition may not seem relevant to the above argument, consider the conditions upon which Bet 2 is taken. The above Dutch book requires that Bet 2 be given to Beauty "upon awaking", but in the case where the coin lands on heads, this instruction is ambiguous since Beauty wakes up twice. The bookie must only give Bet 2 to Beauty once for the above Dutch book to be a Dutch book, for if two Bet 2 s are made, Beauty makes $\$ 60$ while paying only $\$ 55$ and thus obtains a $\$ 5$ payoff. Therefore, the Dutch bookie must have information that Beauty doesn't (i.e. how many Bet 2s have been offered in the past) when he proposes his bets if he wants to be guaranteed to make money off of Beauty no matter what. Since a Dutch book requires that bookie and book-ee have the same information available to them, the above "diachronic Dutch book" is not actually a Dutch book ${ }^{4}$.

Hitchcock suggests the following consideration so that the above mistake above does not happen again: the bookie in the sleeping beauty problem should go to sleep at the same time Beauty does, be awakened when Beauty is awakened, and be administered the same drugs Beauty takes. If these conditions are respected, the bookie will not have any information Beauty lacks. Hitchcock provides a new Dutch book argument that respects this consideration, but his argument is now against the halfer. Consider the following new bets:

Bet $1^{\prime}$ (before sleep): $\$ 30$ if heads, nothing if tails. Cost: $\$ 15$

Bet $2^{\prime}$ (upon each awaking): $\$ 20$ if tails, nothing if heads. Cost: $\$ 10$

\footnotetext{
${ }^{3}$ Such a condition must be imposed so that the Bayesian updating of credences is not found incoherent: if a paleontologist has some reasonable credence $\mathrm{x}$ that the dinosaurs were destroyed by a meteor, and if there is, unbeknownst to him, a dinosaur skeleton yet to be discovered which would lead him to rationally update his credence to y upon discovering this skeleton, one would not want to say that his assignment of credences in these cases was somehow incoherent.

${ }^{4}$ One can, in fact, construct a more general proof that no Dutch book may be constructed against the thirder given the Beauty setup. The following is a short sketch of such a proof. Assume, as Hitchcock does, that the second set of bets are now made upon waking up and that the cost of the nth bet is $Q_{n} c_{n}$, where $Q_{n}$ is the payoff for the nth bet and $c_{n}$ is the credence Beauty has in the outcome that awards her $Q_{n}$ (Beauty will take such bets since $Q_{n} c_{n}$ is the fair price for these bets). There are two situations: one in which the coin is heads, and one where it is tails. If the coin flip results in heads, the bookie makes $Q_{1} c_{1}+2 Q_{2} c_{2}-Q_{1}$, while if the coin flip results in tails, the bookie makes $Q_{1} c_{1}+Q_{2} c_{2}-Q_{2}$. For this problem $c_{1}$ is assumed to be $\frac{1}{2}$ and the thirder chooses $c_{2}=\frac{1}{3}$. So, the bookie now makes either $\frac{2}{3} Q_{2}-\frac{1}{2} Q_{1}$ or $\frac{1}{2} Q_{1}-\frac{2}{3} Q_{2}$. Since one of these payoffs is the negation of the other, the bookie can never be assured that he will make money, and thus he cannot Dutch book a thirder Beauty.
} 
These bets are acceptable to Beauty assuming the credences of the halfer. Now, two situations arise involving these bets. Consider first the case where the coin flip is tails: Beauty takes Bet $1^{\prime}$ before falling asleep, then takes Bet $2^{\prime}$ when she awakes, which is only once. She pays $\$ 25$ for the two bets and, because the flip was tails, she makes $\$ 20$. Overall, then, she has lost $\$ 5$. Now, consider the case where the coin flip is heads: Beauty takes Bet $1^{\prime}$ before falling asleep, but since she is awakened twice, she makes Bet $2^{\prime}$ twice. Thus, she pays $\$ 35$ overall for the three bets, but, because only Bet $1^{\prime}$ provides her with any payoff, she only makes $\$ 30$. Again, Beauty loses $\$ 5$. Since Beauty loses $\$ 5$ no matter what, and since the bookie has no more information than Beauty has when he proposes these bets, the above bets constitutes a Dutch book against the halfer.

The fact that a Dutch book can be made against a halfer Beauty but not a thirder Beauty gives one a reason to prefer the thirder solution to the traditional sleeping beauty problem, but can it help with the Qeauty case? The cases are, after all, supposedly analogous, according to Lewis. I argue that no Dutch book can be made against a halfer Qeauty, and thus Hitchcock's result for the Beauty case does not carry over to the Qeauty case. Let's examine the Dutch book argument against the halfer in the Beauty case to see if the same argument holds in the Qeauty case; if so, it would seem that Lewis's condemnation of the Everettian for her halfer solution is unjustified. Again, examine the following two bets:

Bet $1^{\prime}$ (before sleep): $\$ 30$ if heads, nothing if tails. Cost: $\$ 15$

Bet $2^{\prime}$ (upon each awaking): $\$ 20$ if tails, nothing if heads. Cost: $\$ 10$

If Qeauty is a halfer, she accepts both of these bets when they are offered. Now, assume that the coin flip lands on tails. Qeauty takes Bet $1^{\prime}$ and Bet $2^{\prime}$ once each. She pays $\$ 25$ for the two bets combined and only makes $\$ 20$ from Bet $2^{\prime}$, so she loses $\$ 5$ overall. The situation involving a heads on the coin flip is a bit more complicated. There are two possibilities should the coin land on heads: the spin-measurement following the coin flip could yield an up or down spin result corresponding to a branch in which Qeauty is awakened on Monday or a branch where Qeauty is awakened on Tuesday respectively. Consider the first branch: the coin flip is heads on this branch, and the spin measurement is up. Qeauty's successor is awakened only once on this branch, so, as in the tails case, she takes Bet $1^{\prime}$ and Bet $2^{\prime}$ once each. She pays $\$ 25$ for the two bets combined but gets a payoff of $\$ 30$, thus gaining $\$ 5$. The down measurement case is the same: Qeauty's successor is only awakened once, so she takes both bets only once and ends up making $\$ 5$. Since Qeauty makes $\$ 5$ if the coin lands on heads (regardless of the spin measurement outcome) and loses $\$ 5$ if the coin lands on tails given this series of bets, these bets do not constitute a Dutch book ${ }^{5}$.

\footnotetext{
${ }^{5}$ One can, in fact, construct a more general proof that no Dutch book may be constructed against the halfer given the Qeauty setup. This proof follows along the lines of the proof given in footnote 4: the bookie will make either $Q_{1} c_{1}+Q_{2} c_{2}-Q_{1}$ or $Q_{1} c_{1}+Q_{2} c_{2}-Q_{2}$. If $c_{1}$ is $\frac{1}{2}$ and the halfer takes $c_{2}$ to be $\frac{1}{2}$, then the payoff is either $\frac{1}{2} Q_{2}-\frac{1}{2} Q_{1}$ or $\frac{1}{2} Q_{1}-\frac{1}{2} Q_{2}$. Since one payoff is negative if the other is positive, the bookie cannot be assured that he will make money off of a halfer Qeauty, and so the halfer Qeauty cannot be Dutch booked.
} 
Now, consider the original bets the Hitchcock proposed as a "Dutch book" against the thirder Beauty:

Bet 1 (before sleep): $\$ 30$ if tails, nothing if heads. Cost: $\$ 15$

Bet 2 (upon awaking): $\$ 30$ if heads, nothing if tails. Cost: $\$ 20$

These bets did not constitute a diachronic Dutch book against the thirder because Beauty was awakened twice if the coin flip came out heads, meaning that the bookie would have to know that Bet 2 had not been previously offered to Beauty for this series of bets to constitute a Dutch book. Such information went beyond what was available to Beauty, but in Qeauty's case, the analogous information needed would be that Bet 2 has not been offered to Qeauty at any point in the past along any individual branch. This information is available to Qeauty and her bookie both since each know that there is only one awakening along any individual path. Let's examine this point in more detail. Consider the case in which tails is flipped. In this case, Qeauty is awakened only once, as is the bookie, and so Qeauty loses $\$ 5$ if she has accepted both Bet 1 and Bet 2 (she has paid $\$ 35$ and won only $\$ 30$ ). Consider now the case in which heads is flipped and the measurement yields a value of spin-up. In this history, there has been no Bet 2 offered to Qeauty, and so when the bookie and Qeauty are awakened, he offers her Bet 2 and she accepts, meaning that Qeauty loses $\$ 5$ (she has paid $\$ 35$ and won only $\$ 30$ ). Likewise, the bookie's successor in the spin-down world has never offered Qeauty Bet 2 before, so the bookie makes $\$ 5$ on this branch. Qeauty is not awakened again on any of these branches, and thus, no matter what the coin flip result or the spin measurement result, Qeauty's successors are awakened only once, meaning that the bookie needs no extra information about which world (or branch) he is in to be able to propose the Dutch book. Bets 1 and 2 constitute a Dutch book for Qeauty since the bookie is placed in the same epistemic situation as Qeauty and can offer her a series of bets she would accept which always lead to her losing money.

What I have shown in this section is the following: in the Beauty case, one has a reason to prefer the thirder solution since a diachronic Dutch book can be constructed against the halfer but not against the thirder; in the Qeauty case, however, one has a reason to prefer the halfer solution since a diachronic Dutch book can be constructed against the thirder but not the halfer. This disagreement, I believe, suggests that there is some important difference between the Beauty and Qeauty cases that provides the disanalogy Lewis claimed would be fatal to his argument.

\section{The Difference}

To say that the Beauty and Qeauty cases are disanalogous simply because diachronic Dutch books can be made against different positions in these two cases would be too facile. In this section I discuss what differences between the two cases underwrite their Dutch book disagreement. My analysis shows that, in Papineau and Dura-Villa's words, one can be both a thirder (with respect to the Beauty case) and an Everettian.

I start my analysis again with Hitchcock's "Beauty and the Bets" paper, where he identifies the crucial feature of the Beauty setup that leads to the Dutch book 
argument against the halfer: "Thus the actual number of awakenings that occurs in the two different outcomes does play a central role in the solution to the problem" (415). Hitchcock's analysis here seems right; what made Beauty's Dutch book against the halfer work was the fact that one coin flip outcome corresponded to a different number of awakenings than the other coin flip outcome. It is not surprising, then, that Beauty's halfer Dutch book does not succeed in the Qeauty case where each of Qeauty's successors is awoken only once. In the Dutch book against the halfer Beauty, the "heads" case of these bets yields a $\$ 5$ payoff to the bookie because Bet $1^{\prime}$ is made only once while Bet $2^{\prime}$ is made twice. In the Qeauty analogue, however, each bet is made only once.

This difference in the number of awakenings between the cases might seem strange; after all, Lewis has provided grounds on which to expect Everettian branching to behave like taking the forgetfulness drug in the Beauty case. However, what Lewis failed to take into account was that each Everettian branch is causally isolated from the other branches. If Beauty were to get a tattoo after her first awakening after a heads flip, she would have that same tattoo upon her second awakening. However, Qeauty's spin-up successor's actions never affect Qeauty's spin-down successor. There is thus a metaphysical difference between the two cases.

This metaphysical difference between the two cases can be understood in many ways: one could cash out the difference in terms of causality (or at the very least counterfactuals) since Beauty's two heads-successors are causally connected while Qeauty's two heads-successors are causally isolated. One other option would be to express this difference in terms of identity: Beauty's two heads-successors can be understood as "the same person", or Beauty's Tuesday-heads-successor could be thought of as the successor of Beauty's Monday-heads-successor; however, Qeauty's heads-successors are counter-parts and are not properly regarded as identical, nor is either the successor of the other. Finally, one could understand this metaphysical difference in terms of independence of events. Beauty's Monday-waking and Tuesday-waking on the heads branch are not properly independent events: one can happen only if the other happens. However, in the Qeauty cases, the Monday-waking and Tuesday-waking events on the heads branch are as independent from each other as they are from the Tuesday-waking event on the tails branch ${ }^{6}$.

I believe any of these ways of understanding the metaphysical difference between the Beatuy and Qeauty cases is valid, and, depending on one's stance on various issues in metaphysics, several of these differences may be equivalent; however, Lewis asked for a salient epistemological difference between the cases, and so, before ending my discussion, I must provide such a difference. I think the metaphysical differences previously considered are suggestive here. Let's consider the kinds of uncertainty that both Beauty and Qeauty are faced with when they wake up; that is, what kinds of self-locating uncertainty they have to deal with. Beauty is uncertain of what the coin flip result was and what day it is, but she is uncertain of one further thing: whether she's been awakened before. A thirder Beauty assigns a one-third credence to the

\footnotetext{
${ }^{6}$ I do not pretend here that I have fully spelled-out what the metaphysical difference between the Beauty and Qeauty cases consists in, but such detailed exposition was not my goal; rather, I have hopefully provided sufficient (and minimal) metaphysical grounds in which one can base a rejection of Lewis's claim that the Beauty and Qeauty cases should be treated as analogous.
} 
proposition that she has been awakened before. Qeauty, like Beauty, is also uncertain of what day it is and what the coin flip result was (and, what's more, she's even uncertain of the spin-measurement outcome), but she is certain that she has never been awakened before. No matter what branch Qeauty wakes on, she knows that this waking event is both her first and her last for the duration of the experiment. Thus, the fact that Beauty is uncertain about whether she's been awakened before when she awakes indicates an epistemic difference between her case and the case of Qeauty, who knows that she will only be awakened once on each branch.

So what should the Everettian's response to Lewis be? It should be something like this: in the Beauty case, the thirder's position seems correct; however, were the situation to involve not one person waking up twice but two successors being awoken once each, as Everettians take the Qeauty case to involve, the halfer solution would be the correct one. Thus, from the fact that the thirder solution holds in the Beauty case, it does not follow that the thirder solution is correct in the Qeauty case as well.

\section{Conclusion}

Convincing though Lewis's quantum sleeping beauty argument may seem at first, it hinges on the parallels between what I have called the Beauty and Qeauty cases. The parallels that Lewis considers, however, are swamped out by differences between the two cases, which are indicated by the diachronic Dutch books that may be made against a halfer Beauty and a thirder Qeauty. These Dutch books are indicative of various metaphysical and epistemological differences between the two cases and provide ample motivation to reject Lewis's analogy; thus, despite Lewis's claims, the quantum sleeping beauty case does not reveal inconsistencies in the Everettian's treatment of probability ${ }^{7}$.

\section{References}

[1] Arntzenius, F. "Reflections on Sleeping Beauty". Analysis 62.1, January 2002. pp 53-62.

[2] Dorr, C. "Sleeping Beauty: In Defense of Elga". Analysis 62.4, October 2002. pp 292-96.

[3] Elga, A. "Self-locating belief and the Sleeping Beauty problem". Analysis 60.2, April 2000. pp 143-147.

[4] Greaves, H. "Probability in the Everett Interpretation". Philosophy Compass 2(1), January 2007. pp 109-128

[5] Hitchcock, C. "Beauty and the Bets". Synthese 139, 2004. pp 405-420

[6] Lewis, D. "Sleeping Beauty: reply to Elga". Analysis 61.3, July 2001. pp 171-76.

[7] Lewis, P. "Quantum Sleeping Beauty". Analysis 67.1, January 2007. pp 59-65

\footnotetext{
${ }^{7}$ I would like to thank David Baker, Wayne Myrvold, Laura Ruetsche, Gordon Belot, Dmitri Gallow, Samuel Fletcher, and the audience at the University of Western Ontario's 2009 LMP Graduate Conference for helpful comments on previous drafts of this paper.
} 
[8] Papineau, D. and V. Dura-Vila. "A Thirder and an Everettian: a reply to Lewis's Quantum Sleeping Beauty"'. Analysis Advance Access published on January 1, 2009, DOI 10.1093/analys/ann012. Analysis 69: 78-86. 